\title{
Le lien confédéral: quelques réflexions critiques méthodologiques sur un questionnaire
}

\author{
"Vraisemblance, mais point de vérité: apparence \\ de liberté, mais pas de vérité - c'est à cause \\ de ces deux fruits que l'Arbre de la Science ne \\ risque pas d'être confondu avec l'Arbre de la \\ vie." \\ F. Nietszsche
}

Je n'ai pas l'intention d'ajouter quelques pages à la montagne d'écrits traitant de la diversité linguistique de la Suisse et de son dysfonctionnement éventuel, ni même d'entonner un couplet revendicateur quelconque. Certes, le sujet abordé par le questionnaire dont il est ici question est actuellement d'un intérêt manifeste, mais les mêmes remarques méthodologiques qui font l'objet de cette communication auraient également pu se rapporter à l'étude des habitudes alimentaires des Papous de Nouvelle Guinée...

En 1984, on m'a chargé de dépouiller les résultats d'une enquête réalisée par la Société Suisse d'Utilité Publique. Avec pour titre "Le lien confédéral", ce questionnaire traite des relations entre les différentes communautés linguistiques de la suisse. L'échantillon auquel l'enquête a été soumise est constitué des 1300 officiers qui ont suivi les cours des écoles centrales de l'armée suisse en 1984. Trois points ont retenu mon attention: la conception du questionnaire, la représentativité de la population touchée et le but du questionnaire.

\section{Conception du questionnaire}

Le premier obstacle auquel je me heurtai fut la difficulté, voire l'impossibilité de codifier ce questionnaire afin de le traiter au moyen des logiciels disponibles sur l'ordinateur de l'Université. Il fallut donc procéder à une réinterprétation des questions avant même de songer au dépouillement: certes, on ne peut pas parler de problèmes insurmontables, mais ranger ces complication dans la catégorie des aléas inhérents à toute recherche. En revanche, des problèmes d'interprétation sont rapidement surve- nus: comment préparer le "questionnement" des résultats lorsqu'on ne connait pas le dessein des auteurs. Je ne savais qu'une chose: la Société Suisse d'Utilité publique s'était rendu compte que l'équilibre entre les communautés linguistiques n'était pas ce qu'il aurait dû être; elle estimait important de connaitre l'avis de quelques Suisses pour en faire état et, par conséquent, imaginer des solutions avant que se produise un malaise irréversible. Pour généreux que soit le thème abordé, il manque néanmoins de substance pour permettre une analyse rigoureuse des faits.

" Ce qui se conçoit bien s'énonce clairement

Et les mots pour le dire arrivent aisément." Les auteurs de ce texte ne s'inspirent pas de Boileau. Des imprécisions fleurissent; elles ont probablement perturbé les officiers qui répondent à certaines questions de manière peu cohérente. Prenons les questions 18, 19 et 20. A qui sont-elles adressées ? Fautil comprendre qu'on ne répond qu'aux questions se rapportant aux langues qui ne sont pas les siennes ? Ou alors la précision "per partecipanti di lingua materna italiana" signifie-t-elle que seuls les Suisses italiens ont le droit de répondre ? Peu clair. Même si ce n'est pas vraiment logique, plusieurs personnes y ont répondu.

Les questions 12,13 et 14 prêtent également à confusion. De quelles régions s'agit-il? Sont-elles limitées au territoire national ? La région est une notion imprécise, il n'existe pas de définition univoque. Suivant les auteurs, elle désigne une zone de quelques kilomètres carrés: "la regio genevensis" ou d'une dimension plus considérable: la région des Grands Lacs. Le bon sens ou simplement le jugement des personnes interrogées ne devrait pas suppléer au manque de rigueur des auteurs: d'aucuns étendent la notion "d'autres régions linguistiques" à l'étranger, d'autres

Claire Fischer, licenciée en géographie. Département de droit constitutionnel, Université de Genève. 
la restreignent au territoire national. La question fermée est porteuse d'une information pauvre, des pourcentages. Dans ce cas (questions 12, 13, 14), il devient illusoire d'utiliser des résultats à ce point approximatif.

Quelle est l'intention cachée derrière ces questions ? Il aurait été plus intéressant de déterminer le rôle des médias dans la formation de l'opinion publique, leur origine géographique, leur couleur politique, leur orientation confessionelle ou lä̈que. Quelle est la signification d'un taux d'écoute de la DRS de $30 \%$ de francophones? Quelles émissions suivent-ils ? Il n'est pas nécessaire d'avoir une connaissance approfondie de la langue allemande pour suivre un concert, une émission de variétés ou un match de football. De même, on peut lire "Bilanz", et se désintéresser de l'activité artistique OutreSarine. Que ce journal soit zurichois ne sera certainement pas le facteur dominant dans la décision de l'acheter. Son orientation et le fait qu'il n'existe pas d'équivalent en Suisse Romande constituent des facteurs décisifs dans le choix de certains hommes d'affaires.

\section{Représentativité de la population} touchée par l'enquête

Le choix des sujets interrogés est-il judicieux ? De toute évidence, il n'est pas fortuit. Certes "dans nos cantons, chaque enfant naît soldat" comme le disait Amiel (Roulez, Tambours). L'armée suisse est selon la constitution une armée de milice. J'ai remarqué qu'une grande partie des officiers a obtenu, sinon un diplôme universitaire, la maturité ou tout au moins a terminé une formation complémentaire. Quasiment pas d'employés subalternes, quelques paysans, beaucoup de directeurs, de cadres ou de foncionnaires composent l'échantillon. En vertu de quels critères peuton justifier qu'il est représentatif de la population helvétique? Les femmes, les simples soldats et les retraités disposent également d'une faculté de jugement et il est regrettable de les avoir laissés pour compte. Bien sâr, il est plus commode "d'utiliser" ce rassemblement d'hommes disposés à réaliser ce qu'on leur demande que de chercher à atteindre des citoyens répartis sur le territoire.

A l'instar de tout corps constitué, une discipline militaire aboutit parfois à une uniformisation et à un nivellement des personnalités. Le contexte dans lequel une recherche est menée est extrêmement important; je doute qu'une salle de théorie d'école centrale soit le lieu propice à une réflexion personnelle et indépendante. Non que je soupçonne leurs supérieurs hiérarchiques de brimer ceux qui ne répondraient pas "correctement", mais davantage parce que les officiers n'avaient de fait pas d'autre issue que de remplir les quatre pages. Peu importe comment, mais il y a une pression institutionnelle implicite pour que chacun réponde au questionnaire dès lors que celui-ci est distribué et récolté dans uns salle de classe (et à plus forte raison dans une école centrale). En conséquence, le taux de réponse sera très élevé; situation idéale pour le chercheur mais est-ce là une situation conforme à la réalité ?

Certes, ce questionnaire a été administré à des militaires originaires de l'ensemble de la suisse et, par conséquent de toutes les régions linguistiques; pourtant, il est rédigé en deux langues seulement: l'allemand et le français. Passe encore que le romanche ait été omis, l'administration fédérale elle-même ne traduit que rarement les documents officiels en romanche. Mais négliger la traduction italienne est maladroit; en effet, dans la constitution helvétique, l'italien a le statut de langue officielle: soit les rédacteurs postulent que les italophones comprennent tous l'allemand ou le français, soit ils considèrent ces derniers comme quantité négligeable et jugent préférable d'économiser une traduction en italien. Cette lacune fut très mal perçue par certains "répondants" - et pas seulement par les ressortissants suisses italiens - qui se sont permis de douter de la crédibilité d'une enquête à ce point lacunaire.

\section{Buts ... et lacunes de l'enquête}

Pour en terminer avec cette lecture critique, j'aimerais souligner certaines lacunes de ce questionnaire qui me semblent témoigner d'une vision mythique de la Suisse, sans grand rapport avec la réalité.

Il eat été pertinent de s'intéresser à la pratique de la langue anglaise. Qu'on le veuille ou non, "les espaces concrets et abstraits investis par l'anglais augmentent de même que les durées d'utilisation" comme le dit C.RAFFESTIN dans son ouvrage "Pour une géographie du pouvoir" (1980). Certes ce n'est pas demain que nous nous adresserons à l'employé des CFF à Chiasso ou à Romanshorn en anglais; il est indéniable cependant que pour certains de nos compatriotes, même entre eux, l'anglais devient par- 
tie prenante de certaines relations quotidiennes. Pour ne pas quitter la sphère de l'université, il suffit de citer le nombre de publications rédigées en anglais. Pensons aux multinationales dans lesquelles plus personne ne songerait à mettre en question la pratique généralisée de la langue de Shakespeare. Anecdote vraie: deux sous-officiers convoqués à un cours de répétition "mixte" (suisses allemands, romands et suisses italiens) afin de fonctionner comme secrétaires avaient pour unique véhiculaire l'anglais, d'où incompréhension d'un gradé choqué par ces pratiques.

A la simple lecture de ce questionnaire, on remarque que tout ce qui est étranger à la Suisse n'existe simplement pas ou alors est assimilé à une catégorie fourre-tout "autre". L'image du lien confédéral apparât alors comme un ensemble de relations qui se jouent dans un système clos et replié sur lui même. A l'heure des multinationales, alors que la Suisse est une plaque tournante du système financier international, une telle représentation est fâcheusement naîve, voire mystificatrice et s'apparente dangereusement au concept éminemment militaire de "réduit national". Et pourtant, on ne peut nier l'importance des relations, même souvent conflictuelles avec les pays voisins, que ce soit sur le plan culturel qu'économique ou politique. Il suffit de penser qu'un géographe romand n'aura des pratiques et des publications de ses compatriotes alémaniques qu'une connaissance moindre que celle qu'il peut avoir de la production géographique française. C'est certainement regrettable, mais c'est indéniable... Dans ces conditions, pourquoi nier l'enrichissement apporté par l'extérieur?

De même, ce questionnaire n'effleure pas les problèmes économiques; si souvent les revendications s'expriment sur un plan linguistique, la tension primordiale se réalise sur le plan économique: dans le phénomène de concentration du pouvoir économique dans le "triangle d'or", l'aspect linguistique ne joue qu'un rôle secondaire; d'ailleurs, comme je le soulignais déjà, les affaires se traitent en anglais....

Plutôt que de tâtonner sur une notion aussi creuse et polysémique que celle de lien confédéral, il serait préférable de réfléchir au moyen de rendre les communautés aptes à prendre en main leur destin; à quoi bon lutter pour la sauvegarde d'une langue si ses locuteurs doivent recourir à un autre idiome pour la plupart de leurs relations quotidiennes ? L'intérêt du fédéralisme helvétique tient à la coexistence et la cohabitation entre des cultures vivantes différentes, même si ces relations sont souvent difficiles.

\section{EIDGENÖSSISCHES VERSTÄNDNIS / LIEN CONFÉDÉRAL}

\section{Fragebogen an die Teilnehmer der Zentralschulen 1984 der Armee}

Questionnaire aux participants des écoles centrales 1984 de l'armée

1. Zentralschule / Ecole centrale:

2. Alter / Age:

3. Muttersprache / Langue maternelle:

4. Muttersprache Ihrer Gemahlin oder Lebensgefährtin : Langue maternelle de votre épouse ou compagne:

5. In welchem Kanton wohnen Sie? Dans quel canton résidez-vous?

6. Seit wievielen Jahren? / Depuis combien d'années?

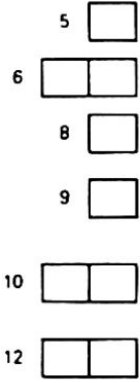

7. Schulische Ausbildung / Formation scolaire:

Sekundarschule Ecole secondaire
Berufsschule / Ergänzungsbildung Ecole professionnelle / Formation complémentaire
Uni / Hochschule Uni / Haute école

8. Erlernter Beruf / Erworbener Titel: Profession apprise / Diplôme obtenu: 
9. Stellung im Beruf / Situation professionnelle:

$\begin{array}{cccccc}\begin{array}{c}\text { selbständig } \\ \text { indépendant }\end{array} & \begin{array}{c}\text { freier Beruf } \\ \text { profession libérale }\end{array} & \begin{array}{c}\text { Manager, Kader } \\ \text { directeur, cadre }\end{array} & \begin{array}{c}\text { Angestellter } \\ \text { employé }\end{array} & \begin{array}{c}\text { Beamter } \\ \text { fonctionnaire }\end{array} & \begin{array}{c}\text { Arbeiter } \\ \text { ouvrier }\end{array} \\ \square & \square & \square & & \end{array}$

10. Gesprochene Sprachen / Langues parlées:

10.1 fliessend / couramment

$$
\text { Schwyzerdütsch }
$$

ziemlich gut / assez bien

schlecht / mal

gar nicht / pas du tout

10.2 gerne / volontiers

widerwillig / à contre-cœur

10.3 gelernt / appris

$$
\begin{aligned}
& \text { in der Familie / en famille } \\
& \text { in der Schule / à l'école } \\
& \text { während Aufenthalten } \\
& \text { au cours de séjours } \\
& \text { durch Kontakte } \\
& \text { par des contacts }
\end{aligned}
$$

11. Verstandene Sprachen / Langues comprises

$$
\begin{aligned}
& \text { beim Lesen / à la lecture } \\
& \text { auf der Strasse / dans la rue } \\
& \text { im Gespräch / en conversation } \\
& \text { Radio, Fernsehen / radio, TV } \\
& \text { Vortrag / conférence } \\
& \text { gar nicht / pas du tout }
\end{aligned}
$$

12. Verfolgen Sie die Radio/TV Programme anderer Sprachregionen? Suivez-vous les programmes radio/TV d'autres régions?

$\begin{array}{cc}\text { Radio } & \text { TV } \\ \square & \square \\ \square & \square \\ \square & \square \\ \square & \square\end{array}$

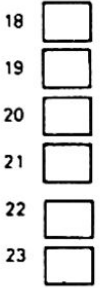

$$
\text { regelmässig / régulièrement }
$$

of / souvent

gelegentlich / partois

nie / jamais

13. Welche Programme? / Que/s programmes?

Suisse romande

DRS

Ticino

Ausland / étranger

\begin{tabular}{|c|c|c|c|}
\hline $\begin{array}{l}\text { familiäre } \\
\text { de famille }\end{array}$ & $\begin{array}{c}\text { berufliche } \\
\text { professionnels }\end{array}$ & $\begin{array}{l}\text { Freizeit } \\
\text { loisirs }\end{array}$ & $\begin{array}{l}\text { andere } \\
\text { autres }\end{array}$ \\
\hline
\end{tabular}

14. Haben Sie Beziehungen zu Personen der anderen Sprachregionen?

Avez-vous des liens avec des personnes d'autres régions linguistiques?

Welche Regionen / Quelles régions?

15. Gehören Sie Vereinen an, die Mitglieder in verschiedenen Sprachregionen haben?

Faites-vous partie d'associations groupant des participants de différentes régions linguistiques?

$\begin{array}{ll}\text { ja } & \text { nein } \\ \text { oui } & \text { non }\end{array}$

16. Arbeiten Sie aktiv mit? / Y participez-vous activement? 
17. Haben Sie sprachliche Schwierigkeiten?

Avez-vous des difficultés linguistiques?

\begin{abstract}
bei ihren Zusammenkūnften lors de leurs réunions

bei der Lektüre ihrer Dokumente

a la lecture de leurs documents
\end{abstract}

18. Welche Unterschiede stellen Sie bei Personen der Welschen Schweiz fest? Quelles différences percevez-vous avec des personnes de Suisse romande? (per partecipanti di lingua materna italiana)

$\begin{array}{lcccc} & \begin{array}{c}\text { erhebliche } \\ \text { considérables }\end{array} & \begin{array}{c}\text { grosse } \\ \text { grandes }\end{array} & \begin{array}{c}\text { geringe } \\ \text { pétites }\end{array} & \begin{array}{c}\text { keine } \\ \text { aucune }\end{array} \\ \text { Charakter / caractère } & \square & \square & \square & \square \\ \text { Handlungsweise / manière d'agir } & \square & \square & \square & \square \\ \text { Meinungen / opinions } & \square & \square & \square & \square \\ \text { andere (welche) / autres (lesquelles) } & \square & \square & \square\end{array}$

19. Welche Unterschiede stellen Sie bei Personen der Italienischen Schweiz fest? Quelles différences percevez-vous avec des personnes de Suisse italienne?

\begin{tabular}{lcccc} 
& $\begin{array}{c}\text { erhebliche } \\
\text { considérables }\end{array}$ & $\begin{array}{c}\text { grosse } \\
\text { grandes }\end{array}$ & $\begin{array}{c}\text { geringe } \\
\text { petites }\end{array}$ & $\begin{array}{c}\text { keine } \\
\text { aucune }\end{array}$ \\
Charakter / caractère & $\square$ & $\square$ & $\square$ \\
Handlungsweise / manière d'agir & $\square$ & $\square$ & $\square$ \\
Meinungen / opinions & $\square$ & $\square$ & $\square$ \\
andere (welche) / autres (lesquelles) & $\square$ & $\square$ & $\square$ \\
\hline$\square$
\end{tabular}

20. Welche Unterschiede stellen Sie bei Personen der Deutschen Schweiz fest? Quelles différences percevez-vous avec des personnes de Suisse alémanique? (per partecipanti di lingua materna italiana)

$\begin{array}{lccc}\text { erhebliche } & \text { grosse } & \begin{array}{c}\text { geringe } \\ \text { petites }\end{array} & \begin{array}{c}\text { keine } \\ \text { aucune }\end{array} \\ \text { Charakter / caractère } & \square & \square & \square \\ \text { Handlungsweise / manière d'agir } & \square & \square & \square \\ \text { Meinungen / opinions } & \square & \square & \square \\ \text { andere (welche) / autres (lesquelles) } & \square & \square & \square\end{array}$

21. Sind Sie der Meinung, es bestünden... Missverständnisse zwischen den Angehörigen der verschiedenen Sprachregionen der Schweiz? Estimez-vous qu'il existe des malentendus... entre ressortissants des différentes régions linguistiques de Suisse?

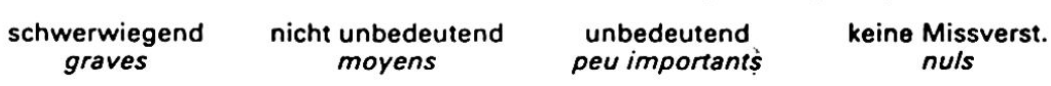
$\square \square \square \square$

Welche Art von Missverständnissen:

Sur quels points portent ces malentendus?

22. Sind diese Missverständnisse, wenn sie bestehen, bedeutender oder unbedeutender als jene, die zwischen den Kantonen der gleichen Sprachregionen bestehen?

Ces malentendus, s'ils existent, sont-ils plus ou moins importants que ceux qui existent entre cantons d'une méme région linguistique?

$$
\begin{gathered}
\text { bedeutender } \\
\text { plus importants } \\
\text { moins importants }
\end{gathered}
$$$$
\square
$$$$
\square
$$ 
23. Sind die öffentlichen und privaten Einrichtungen in den Kantonen fähig, solche möglichen Missverständnisse zu beheben?

Les organisations cantonales publiques et privées sont-elles en mesure de remédier à ces malentendus éventuels?

$\begin{array}{lll}\text { ja } & \text { nein } & \text { ohne Meinung } \\ \text { oui } & \text { non } & \text { sans opinion }\end{array}$

24. Worauf führen Sie die Missverständnisse zurück, die Sie möglicherweise zwischen Sprachregionen entdeckt haben? A quoi attribuez-vous les malentendus que vous avez éventuellement décelés entre régions linguistiques?

\begin{tabular}{|c|c|c|}
\hline $\begin{array}{c}\text { Unkenntnis } \\
\text { méconnaissance }\end{array}$ & $\begin{array}{l}\text { Misstrauen } \\
\text { méfiance }\end{array}$ & $\begin{array}{l}\text { Unverständnis } \\
\text { incompréhension }\end{array}$ \\
\hline$\square$ & $\square$ & $\square$ \\
\hline $\begin{array}{l}\text { Vorurteile } \\
\text { préjugés }\end{array}$ & $\begin{array}{l}\text { Gefühl der } \\
\text { Überlegenheit } \\
\text { complexe de } \\
\text { supériorité }\end{array}$ & $\begin{array}{l}\text { Minderwertig- } \\
\text { keitskomplex } \\
\text { complexe } \\
\text { d'infériorité }\end{array}$ \\
\hline
\end{tabular}

$\square$

25. Denken Sie, dass die Missverständnisse, wenn sie bestehen:

Pensez-vous que les malentendus, s'ils existent, ont des conséquences:

\begin{tabular}{|c|c|c|c|c|}
\hline & $\begin{array}{l}\text { tiefgreifende } \\
\text { profondes }\end{array}$ & $\begin{array}{l}\text { ernsthafte } \\
\text { sérieuses }\end{array}$ & $\begin{array}{l}\text { geringe } \\
\text { légères }\end{array}$ & Auswirkungen \\
\hline $\begin{array}{l}\text { auf die Politik in der Schweiz } \\
\text { sur la politique en Suisse }\end{array}$ & $\square$ & $\square$ & $\square$ & \\
\hline $\begin{array}{l}\text { auf die sozialen Beziehungen } \\
\text { in der Schweiz } \\
\text { sur les rolations sociales } \\
\text { en Suisse }\end{array}$ & $\square$ & $\square$ & $\square$ & haben \\
\hline
\end{tabular}

en Suisse

26. Glauben Sie, dass über Ihre Sprachregion Urteile geäussert werden, die... sind?

Pensez-vous que votre région linguistique est l'objet de jugements?

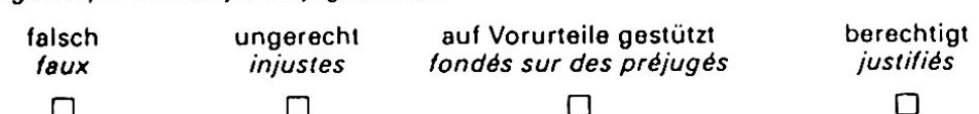

27. Welche Urteile?

Quels jugements?

28. Was schlagen Sie gegen das Malaise vor, das Sie vielleicht empfunden haben?

Quels remedes proposez-vous au malaise que vous avez peut-étre ressenti?

\section{Sprachkurse \\ cours de langues}

Unterricht in Schweizergeschichte

leçons d'histoire suisse

Ferienaufenthalte in einer andern Sprachregion

sejours dans une autre région linguistique

Ausbildungsaufenthalte in einer andern Sprachregion

stages dans une autre région linguistique

Austausch von Schūlern

Echange d'elèves

Austausch von Lehrlingen

Echange d'apprentis

Austausch von Berufsleuten

Echange de travailleurs

touristische Propaganda

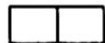

andere, welche?

autres, lesquels? 